\title{
Mouse Model of Tuberculosis
}

\author{
Andrea M. Cooper \\ Trudeau Institute, Inc., Saranac Lake, New York 12983 \\ Correspondence: acooper@trudeauinstitute.org
}

The mouse provides a tool with which to probe the complex interaction between the mammalian immune system and the slow-growing, inflammatory, and persistent bacterium, Mycobacterium tuberculosis (Mtb). Simple mouse models using genetic deletion or antibody inhibition have identified causal connections between specific components of the immune response and survival upon challenge with $M t b$, and these studies have corresponded with observations made in humans. To improve on current intervention strategies, it is essential that the complex interactions between the components of the immune response that mediate and regulate the protective response to $M t b$ infection be dissected; furthermore, the pathways by which specific molecules and cells act must be delineated. The mouse model provides a tool with which to achieve this goal; however, experimental design and data interpretation must be made in the context of data sets generated from the entire tuberculosis field.

$\mathrm{O}_{\mathrm{s}}^{\mathrm{r}}$ rganisms have increasing levels of complexity and when they interact, the level of complexity increases dramatically. Vertebrates have developed the ability to respond to exposure to lower-order organisms in the form of a targeted and highly regulated immune response. The immune response has simple elements at its base including recognition of "danger," recognition of nonself, the ability to adapt to novel antigens, the ability to remember previous antigens, and active regulation of the above responses. When these simple elements act in concert, the host response is immensely adaptable and capable of dealing with a vast array of potential pathogens. Of course, this ability can also lead to inappropriate responses, which contribute to damage and disease. Animal models have been used to identify definitive causal relationships between specific compo- nents of this complex immune response and the survival of the host following exposure to a variety of pathogens. Although these studies remain valid, the complexities of the pathways by which specific components of the immune response interact both to control the pathogen and to limit immunopathology are just now being fully realized. Defining the role of specific factors within complex pathways now requires highly refined and manipulatable tools, and recent developments within the mouse model provide these tools.

Within the context of mycobacterial disease, and tuberculosis in particular, the complexity arising from the persistence of this pathogen and the need for balance between protection and pathogenesis is at the core of our difficulty in controlling this pathogen. The cell types that contribute to the immunity to TB include my-

Editors: Stefan H.E. Kaufmann, Eric J. Rubin, and Alimuddin Zumla

Additional Perspectives on Tuberculosis available at www.perspectivesinmedicine.org

Copyright (C) 2015 Cold Spring Harbor Laboratory Press; all rights reserved; doi: 101101/cshperspect.a018556

Cite this article as Cold Spring Harb Perspect Med 2015;5:a018556 
A.M. Cooper

eloid and lymphoid cells but within these broad groupings are a wide range of cell phenotypes, the importance of which depend on location of the challenge, the nature of challenge, and the constituents of the microbiome, which play a large part in regulating the tonic state of the immune system (Erturk-Hasdemir et al. 2013). To deconvolute the complexity of the immune system and to drill down through the mechanisms that mediate its initiation, expansion, and regulation, it is critical to use a model wherein many of the variables can be controlled. The mouse does not provide a direct mimic of the human response to mycobacterial infection. It does, however, provide a living, breathing vertebrate lung on a small scale with the capacity to perform a large number of experiments with definitive outcomes. It also provides a manipulatable tool that represents the complexity of the vertebrate immune response to Mycobacterium tuberculosis $(M t b)$ while allowing definitive experimentation to isolate the critical components involved in both protection and pathogenesis. The recent increase in tools for the mouse model has allowed us to better define the interaction between the pathogen and the different components of the immune response, and the outcomes have been surprising.

\section{GENETIC APPROACHES}

A powerful tool provided by the mouse is the ability to investigate complex genetic traits in a mammalian model. Mutant mouse strains can be used for "reverse" genetic approaches to query the role of a specific gene in the development of mycobacterial disease in the vertebrate host; this is almost entirely performed with murine models at the current time. "Forward" genetics use random mutation as well as the comparison of inbred strains of mice, which differ in their susceptibility to mycobacterial disease to probe for novel genes in an unbiased manner (Fortin et al. 2007). The variety of susceptibility within inbred mouse strains is highlighted by the fact that $\mathrm{CBA}, \mathrm{C} 3 \mathrm{HeB} / \mathrm{FeJ}, \mathrm{DBA} / 2$, and $129 \mathrm{SvJ}$ are susceptible to $M t b$, whereas $\mathrm{C} 57 \mathrm{BL} / 6 \mathrm{~J}$ and $\mathrm{BALB} / \mathrm{c}$ are resistant (Medina et al. 1998). By crossing the inbred strains and generating con- genic lines, loci associated with the susceptible phenotype have been identified. The use of this tool has resulted in the determination of several independent loci responsible for susceptibility such as sst 1 , which contains the Ipr 1 gene (Pan et al. 2005); loci on chromosomes 9 and 3, which mediate interstrain differences between susceptible I/St and resistant A/Sn strains (Sánchez et al. 2003); and the Trl1-4 loci, which contribute to susceptibility of the DBA/2 J strain (Marquis et al. 2009). Importantly these susceptibility loci are associated with different arms of the protective response and highlight the importance of the balanced integration of the lymphoid, myeloid, and subsequent inflammatory response in maintaining control of $M t b$ in vivo.

\section{TARGETED CELLULAR APPROACHES}

Early events occurring during infection are difficult to model and analyze even in the mouse model. It is thought that aerosol delivery of a suspension of bacteria at a low concentration mimics natural infection, and indeed most of the animal models use this or a variation of this delivery system. Whether this is a true mimic is not clear, as the nature of infectious bacteria arriving from the transmitting individual to the exposed individual is not yet defined. Using this low-dose model, however, results in very few early events and renders investigation of these events difficult. Indirect measures have therefore been used within the mouse model to investigate the importance of specific cell types in the early initiation of the acquired cellular response, which is critical for control of $M t b$. One such model includes genetically modified mice that allow for the timed depletion of specific cells, which express the diphtheria toxin receptor (DTR) (Saito et al. 2001). Recently the use of this model to deplete cells positive for $\mathrm{C}-\mathrm{C}$ chemokine receptor 2 (CCR2) resulted in the surprising observation that inflammatory monocytes (which express CCR2) are required to deliver invading bacteria to the draining lymph node (Samstein et al. 2013). Dendritic cells had been thought to be responsible for this transport, and indeed they appear to act in this capacity once infection is established (Wolf et al. 
2007); however, the inflammatory monocytes, although responsible for delivery, were not responsible for driving T-cell activation (Samstein et al. 2013). Using the CD11c ${ }^{+}$DTR model (Jung et al. 2002), it was found that $\mathrm{T}$ cells were compromised in their ability to be activated if CD $11 c^{+}$cells lacked the IL-12R $\beta 1$ (Robinson et al. 2010). There are other genetic systems available that can be used for timed depletion of specific cell types or of specific molecules within specific cell types such as the cre/loxP system (Kühn et al. 2002). Concerns with these models that must be considered when using them in the context of $M t b$ infection include the ability of the depleting agent to penetrate the macrophage-dominated lesion and the impact of significant cell death on the integrity of the granulomatous lesion. Despite these concerns, the use of these targeting systems to deplete molecules and cells within a defined time frame and within specific cell types have increased the power of the initial genetic-deletion techniques that were used to confirm the roles of specific components of the immune response such as IFN- $\gamma$, TNF, and IL-12 in controlling $M t b$ infection (Cooper et al. 1993, 1997; Flynn et al. 1993, 1995).

\section{EXAMINING T-CELL RESPONSES WITHIN THE $M t b$-INFECTED MOUSE}

Understanding how the body recognizes invasion by $M t b$ and initiates the expansion of antigen-specific T cells is important in understanding pathogenesis and in vaccine design. Because of the low frequency of initiating events following low-dose aerosol infection, it is necessary to have indirect measures of antigen presentation. In recent studies with the mouse model, one aspect of early infection was highlighted when it became clear that the acquired immune response largely ignores the arrival of the bacterium in the lung following aerosol infection (Chackerian et al. 2002; Reiley et al. 2008; Wolf et al. 2008). In these papers, analyses of antigen-specific cellular responses were made using specific antigens and/or T-cell receptor transgenic (TCRTg) $\mathrm{T}$ cells. The importance of the TCRTg T cells is that they provide a highly sensitive tool with which to indicate the kinetics and location of appropriately presented antigen when delivered exogenously and examined at specific times posttransfer (Kearney et al. 1994). In three separate laboratories the timing of initial naïve T-cell activation was found to be delayed relative to challenge $(\sim 7-9 \mathrm{~d})$ and was found to occur within the lung-draining lymph node and not to any great extent with the lung (Gallegos et al. 2008; Reiley et al. 2008; Wolf et al. 2008). The use of TCRTg cells was critical to this insight as the very low frequency of the endogenous response meant that the earliest events could not be detected directly. Although TCRTg cells represent a powerful tool, they should be used with care as they are an artificial $\mathrm{T}$ cell and do not develop identically to endogenous T cells. It is also critical to keep the transferred precursor level low as it is known that the precursor frequency of naïve T cells impacts the outcome of the immune response (Kearney et al. 1994; Jenkins et al. 2012; Sprent 2013).

The function of $\mathrm{T}$ cells within the inflamed site induced by $M t b$ infection is also a subject that has been investigated using the mouse model. In this respect, use of immunohistochemistry and live imaging has been crucial. Early intravital studies supported the key role of TNF in development of the macrophage matrix of the $M t b$-induced granuloma (Egen et al. 2008) and also showed that T cells within the macrophage-dominated lesion fail to undergo migration arrest and only occasionally secreted cytokine in a targeted manner (Egen et al. 2011). In a different study, using flow-cytometric examination of $\mathrm{T}$-cell function immediately ex vivo, a low level of antigen availability within the granuloma was associated with poor cytokine production in vivo (Bold et al. 2011). These studies, along with those implicating the importance of correct chemokine receptor expression in T-cell function (Slight et al. 2013), support the importance of $\mathrm{T}$-cell location relative to the $M t b$-infected macrophage in mediating the control of bacterial growth. A recent mouse study addressed the importance of direct T-cell interaction with infected macrophages using a bone marrow chimera model. In these chimeras, bone marrow is transferred into an irradi- 
A.M. Cooper

ated host and repopulates the radiosensitive portions of the hematopoietic system (Shizuru et al. 1996). Using donors that differ in the ability to express specific molecules and that can be differentiated by the expression of a congenic marker, the response of these cells can be compared in vivo with the same stimuli and within the same context. Using this tool it was found that within the same host, only those infected macrophages expressing MHC class II showed CD4 T-cell-dependent reduction of bacterial numbers (Srivastava et al. 2013); thus addressing a long-term question of whether direct T-cell macrophage interaction was required for antibacterial activity by the macrophage. This type of bone marrow chimera model is very useful for a range of immunological investigations of the chronic inflammation induced by $M t b$. Key points are that intact and deficient cells are within the same environment and will not undergo rejection over the long term (which transferred cells would undergo). Concerns include the fact that long-lived, nondividing hematopoietic cells (memory T cells, tissue macrophages, etc.) will not be fully removed by irradiation, and this should be considered and controlled for wherever possible.

Full investigation of the function and phenotype of $\mathrm{T}$ cells during mycobacterial disease has been greatly impacted recently by the use of tetramer staining (Altman et al. 1996) and cell sorting. The tetramer stains used to identify a subset of the antigen-specific cells within an endogenous population of responding cells have proven particularly useful in identifying T-cell trends that were not apparent when studying the bulk population of activated T cells. By focusing on $\mathrm{T}$ cells of one antigen specificity, it has been possible to define several subsets of responding cells, such as those expressing markers associated with antigen exposure (e.g., PD-1 and KLRG-1 [Reiley et al. 2010]). Importantly, the addition of cell sorting to the study of T cells from infected mice has allowed the functional capacity of T-cell subsets to be assessed. Thus, when T cells were subsetted based on their expression of PD1 or KLRG-1, they were found to have different abilities to produce cytokine and to proliferate and persist with an infected host (Reiley et al.
2010). The increased use of tetramer stains in human studies to identify and characterize antigen-specific T cells (Lindestam Arlehamn et al. 2013) will allow the work in the mice to undergo even more refinement and allow for observations made in the mouse to be fully translated to the antigen-specific population in humans. This very powerful set of tools is set to transform our understanding of T-cell function within the human.

\section{PATHOLOGIC CONSEQUENCES OF $M t b$ INFECTION IN THE MOUSE}

Using histological techniques, many studies have been made of the pathologic consequences of infection in the mouse lung, and inclusion of this parameter in any mouse model provides important information regarding the health of the lung tissue. Importantly, although increased bacterial burden and decreased granuloma integrity are usually seen together, it is also the case that the granulomatous response develops over time despite little change in bacterial number (Rhoades et al. 1997). A recent development in our understanding of the role of B-cell follicles in immunity was prompted by the repeated observation of these follicles associated with $M t b$-induced inflammation in the mouse. Using mouse models of gene deletion, bone marrow chimeras, and antibody/serum treatment, the role of B cells in $M t b$ infection has been examined. Recently, it has been shown that $\mathrm{B}$ cells serve to regulate the IL-17 response and thereby reduce neutrophil recruitment to the infection site (Kondratieva et al. 2010; Kozakiewicz et al. 2013). Using genetically depleted mice it was also found that loss of the activating receptor $\mathrm{F} c \gamma \mathrm{R}$ compromises control of $\mathrm{Mtb}$ growth, whereas loss of the inhibitory receptor FcyRIIB improves control (Maglione et al. 2008). In contrast, in models where $B$ cells are present but are unable to generate circulating immunoglobulin, they act to inhibit immunity in association with excess IL-10 production (Torrado et al. 2013). Recent work has indicated an association between B-cell follicles in migration of T cells from the blood vessels throughout the inflamed area of the lungs in mice (Khader 
et al. 2009, 2011), and this work was extended to nonhuman primates (NHPs) and humans. It was found that B-cell follicles were in a minority of lesions from active TB patients, and that Bcell follicles were dominant in latently infected relative to actively infected NHPs (Slight et al. 2013). The mouse model was then used to investigate the causative connection between the B-cell follicles and bacterial control (Slight et al. 2013). It was found that although B-cell follicles are associated with control, they are not required for it and that they are a consequence of the appearance of $\mathrm{C}-\mathrm{X}-\mathrm{C}$ chemokine receptor 5 (CXCR5)-expressing CD4 $\mathrm{T}$ cells, which respond to CXCL13 and which act as follicular helper $\mathrm{T}$ cells. The expression of CXCR5 on the $\mathrm{T}$ cells is required to mediate appropriate migration of the activated $\mathrm{T}$ cell from the blood vessel to colocalize with infected phagocytes and thereby mediate macrophage activation (Slight et al. 2013). This work is a fine example of how the use of multiple models allows for the identification of a phenomenon followed by the definition of the pathways by which the phenomenon occurs.

Although activation of infected macrophages requires colocalization with antigen-specific $\mathrm{T}$ cells, these $\mathrm{T}$ cells are also actively regulated by the environment which they create. Although the ability of T cells to undergo migration arrest and mediate antibacterial function is low (see above), they are very able to activate the accumulated macrophages within the $M t b$-induced lesion to generate a highly inflamed site. How T cells function within this site and how the site is regulated have been extensively addressed using mouse models. Original observations using gene-deleted mice indicated that IFN- $\gamma$ and IL-12p40 were essential for control of $M t b$ infection in the mouse model (Cooper et al. 1993, $1997,2002 b)$, and these studies were complementary to the observation in humans that absence of these genes resulted in Mendelian susceptibility to mycobacterial disease (MSMD) (Filipe-Santos et al. 2006). However, the role of IFN- $\gamma$ has been questioned because of the observation that the simple number of IFN- $\gamma$ producing $\mathrm{T}$ cells in the peripheral blood of humans does not strongly correlate with ability to control mycobacterial disease. Recent studies with mice, using gene-deleted and bone marrow chimera models, have shown that IFN- $\gamma$ expression by $\mathrm{T}$ cells acts to limit the accumulation of neutrophils (Pearl et al. 2001; Nandi et al. 2011), and that it also regulates IL-17 activity (Desvignes et al. 2009; Nandi et al. 2011) within the inflamed site. IFN- $\gamma$ also acts directly to limit the accumulation of activated $\mathrm{T}$ cells within the mycobacterial lesion (Gomes et al. 1999; Cooper et al. 2002a; Nandi et al. 2011), and this may contribute to the inability of the vertebrate host to eliminate the infection. One consequence of IFN- $\gamma$ production is the generation of nitric oxide by macrophages within the granuloma. Although nitric oxide is critical to limit growth of $M t b$, it is also known to regulate the pathologic response to mycobacterial infection (Cooper et al. 2002a). In this regard an antibiotic-dependent $M t b$ was recently used to specifically probe the anti-inflammatory activity of nitric oxide. It was found that lymphocytederived IFN- $\gamma$ limited the production of IL-1 and subsequent immunopathology via the induction of nitric oxide-mediated inhibition of the NLRP3 inflammasome (Mishra et al. 2013). Nitric oxide also acts to limit the accumulation of activated T-cell subsets within mycobacterially infected tissue (Pearl et al. 2012).

Although the pathologic response to $M t b$ infection in the mouse lung does not recapitulate the complete set of outcomes seen in diseased human tissue, manipulation of the mouse model can be used to determine the factors involved in initiating and regulating inflammation. Comparative pathology can then be used to identify factors that, when dysregulated, allow for the type of pathology seen in humans.

\section{USE OF THE MOUSE MODEL TO DEVELOP DRUGS AND VACCINES}

Although the real power of the mouse model lies in the delineation of in vivo immunological and pathological pathways, it can also be used as a small animal model to study drugs and vaccines. It is in these studies where overinterpretation can cause problems. It is essential that the investigator ensures that the tool being used will 
A.M. Cooper

answer the question being asked and also that the scientific community interprets the observations made in light of the constraints of the model. Within drug development it is necessary to understand the context of the bacteria within the mouse; thus, if one wants to screen a drug for its ability to target bacteria in a macrophagedominated lesion in the lung within an immunocompetent host, then a B6 mouse infected with $M t b$ via the aerosol route is potentially suitable. If, however, one needs to target bacteria growing within a neutrophil-dominated or necrotic lesion, then one can use a mouse lacking IFN- $\gamma$ production or signaling (Desvignes et al. 2009; Nandi et al. 2011) or an inbred strain such as I/St (Eruslanov et al. 2005) or mice with the sst1-susceptible locus (Pichugin et al. 2009). The above also applies when considering vaccination; it has been shown that the susceptibility profiles of different mouse strains are maintained after vaccination with BCG (Bacillus Calmette-Guérin) (Medina et al. 1999). Thus if one wants to test a vaccine in a model wherein expression of immunity is compromised, then a susceptible mouse strain is the correct one to use. In terms of vaccine development, however, the mouse most effectively represents the capacity to investigate potential mechanisms of protection rather than a tool to decide which vaccines move forward.

\section{CONCLUSION}

The mouse model allows for both definitive and unbiased approaches with which to probe the interaction between $M t b$ and a vertebrate host. It also provides a manipulatable environment that can reflect aspects of the human infection and disease progression. Overinterpretation and inappropriate translation to human disease can lead to issues, but judicious interpretation within the context of the field as a whole can lead to much greater understanding of the pathways mediating pathogenesis in tuberculosis.

\section{ACKNOWLEDGMENTS}

A.M.C. is supported by Trudeau Institute, Inc., National Institutes of Health grants AI69121,
AI46530, and AI73564, and the Bill and Melinda Gates Foundation.

\section{REFERENCES}

Altman J, Moss P, Goulder P, Barouch D, McHeyzer-Williams M, Bell J, McMichael A, Davis M. 1996. Phenotypic analysis of antigen-specific T lymphocytes. Science 274: 94-96.

Bold TD, Banaei N, Wolf AJ, Ernst JD. 2011. Suboptimal activation of antigen-specific $\mathrm{CD}^{+}{ }^{+}$effector cells enables persistence of $M$. tuberculosis in vivo. PLoS Pathog 7: e1002063.

Chackerian A, Alt J, Perera T, Dascher C, SM B. 2002. Dissemination of Mycobacterium tuberculosis is influenced by host factors and precedes the initiation of T-cell immunity. Infect Immun 70: 4501-4509.

Cooper AM, Dalton DK, Stewart TA, Griffin JP, Russell DG, Orme IM. 1993. Disseminated tuberculosis in interferon $\gamma$ gene-disrupted mice. J Exp Med 178: 2243-2247.

Cooper AM, Magram J, Ferrante J, Orme IM. 1997. IL-12 is crucial to the development of protective immunity in mice intravenously infected with Mycobacterium tuberculosis. J Exp Med 186: 39-46.

Cooper AM, Adams LB, Dalton DK, Appelberg R, Ehlers S. 2002a. IFN- $\gamma$ and NO in mycobacterial disease: New jobs for old hands. Trends Microbiol 10: 221-226.

Cooper AM, Kipnis A, Turner J, Magram J, Ferrante J, Orme IM. 2002b. Mice lacking bioactive IL-12 can generate protective, antigen-specific cellular responses to mycobacterial infection only if the IL-12 p40 subunit is present. J Immunol 168: 1322-1327.

Desvignes L, Ernst JD. 2009. Interferon- $\gamma$-responsive nonhematopoietic cells regulate the immune response to $M y$ cobacterium tuberculosis. Immunity 31: 974-985.

Egen J, Rothfuchs A, Feng C, Winter N, Sher A, Germain R. 2008. Macrophage and T cell dynamics during the development and disintegration of mycobacterial granulomas. Immunity 28: 271-284.

Egen JG, Rothfuchs AG, Feng CG, Horwitz MA, Sher A, Germain RN. 2011. Intravital imaging reveals limited antigen presentation and $\mathrm{T}$ cell effector function in mycobacterial granulomas. Immunity 34: 807-819.

Erturk-Hasdemir D, Kasper DL. 2013. Resident commensals shaping immunity. Curr Opin Immunol 25: 450-455.

Eruslanov E, Lyadova I, Kondratieva T, Majorov K, Scheglov I, Orlova M, Apt A. 2005. Neutrophil responses to $M y$ cobacterium tuberculosis infection in genetically susceptible and resistant mice. Infect Immun 73: 1744-1753.

Filipe-Santos O, Bustamante J, Chapgier A, Vogt G, de Beaucoudrey L, Feinberg J, Jouanguy E, Boisson-Dupuis S, Fieschi C, Picard C, et al. 2006. Inborn errors of IL-12/ 23 - and IFN- $\gamma$-mediated immunity: Molecular, cellular, and clinical features. Semin Immunol 18: 347-361.

Flynn JL, Chan J, Triebold KJ, Dalton DK, Stewart TA, Bloom BR. 1993. An essential role for interferon $\gamma$ in resistance to Mycobacterium tuberculosis infection. J Exp Med 178: 2249-2254.

Flynn JL, Goldstein MM, Chan J, Triebold KJ, Pfeffer K, Lowenstein CJ, Schreiber R, Mak TW, Bloom BR. 1995. 
Tumor necrosis factor- $\alpha$ is required in the protective immune response against Mycobacterium tuberculosis in mice. Immunity 2: 561-572.

Fortin A, Abel L, Casanova JL, Gros P. 2007. Host genetics of mycobacterial diseases in mice and men: Forward genetic studies of BCG-osis and tuberculosis. Ann Rev Genom Hum Genet 8: 163-192.

Gallegos A, Pamer E, Glickman M. 2008. Delayed protection by ESAT-6-specific effector $\mathrm{CD}^{+}{ }^{+} \mathrm{T}$ cells after airborne M. tuberculosis infection. J Exp Med 205: 2359-2368.

Gomes MS, Florido M, Pais TF, Appelberg R. 1999. Improved clearance of Mycobacterium avium upon disruption of the inducible nitric oxide synthase gene. J Immunol 162: 6734-6739.

Jenkins MK, Moon JJ. 2012. The role of naive T cell precursor frequency and recruitment in dictating immune response magnitude. J Immunol 188: 4135-4140.

Jung S, Unutmaz D, Wong P, Sano G, De los Santos K, Sparwasser T, Wu S, Vuthoori S, Ko K, Zavala F, et al. 2002. In vivo depletion of CD11 $\mathrm{c}^{+}$dendritic cells abrogates priming of $\mathrm{CD}^{+} \mathrm{T}$ cells by exogenous cell-associated antigens. Immunity 17: 211-220.

Kearney ER, Pape KA, Loh DY, Jenkins MK. 1994. Visualization of peptide-specific $\mathrm{T}$ cell immunity and peripheral tolerance induction in vivo. Immunity 1: 327-339.

Khader S, Rangel-Moreno J, Fountain J, Martino C, Reiley W, Pearl J, Winslow G, Woodland D, Randall T, Cooper A. 2009. In a murine tuberculosis model, the absence of homeostatic chemokines delays granuloma formation and protective immunity. J Immunol 183: 8004-8014.

Khader SA, Guglani L, Rangel-Moreno J, Gopal R, Fallert Junecko BA, Fountain JJ, Martino C, Pearl JE, Tighe M, Lin YY, et al. 2011. IL-23 is required for long-term control of Mycobacterium tuberculosis and B cell follicle formation in the infected lung. J Immunol 187: 5402-5407.

Kondratieva T, Rubakova E, Linge I, Evstifeev V, Majorov K, Apt A. 2010. B cells delay neutrophil migration toward the site of stimulus: Tardiness critical for effective bacillus Calmette-Guérin vaccination against tuberculosis infection in mice. J Immunol 184: 1227-1234.

Kozakiewicz L, Chen Y, Xu J, Wang Y, Dunussi-Joannopoulos K, Ou Q, Flynn JL, Porcelli SA, Jacobs WR Jr, Chan J. 2013. B cells regulate neutrophilia during Mycobacterium tuberculosis infection and BCG vaccination by modulating the interleukin-17 response. PLoS Pathog 9: e1003472.

Kühn R, Torres RM. 2002. Cre/loxP recombination system and gene targeting. In Transgenesis techniques (ed. Clarke A), Vol. 180, pp. 175-204. Springer, New York.

Lindestam Arlehamn CS, Gerasimova A, Mele F, Henderson R, Swann J, Greenbaum JA, Kim Y, Sidney J, James EA, Taplitz R, et al. 2013. Memory T cells in latent Mycobacterium tuberculosis infection are directed against three antigenic islands and largely contained in a $\mathrm{CXCR}^{+}$ $\mathrm{CCR}^{+}$Th1 subset. PLoS Pathog 9: e1003130.

Maglione P, Xu J, Casadevall A, Chan J. 2008. Fcy receptors regulate immune activation and susceptibility during Mycobacterium tuberculosis infection. I Immunol 180: 3329-3338.

Marquis J, Lacourse R, Ryan L, North R, Gros P. 2009. Genetic and functional characterization of the mouse Trl3 locus in defense against tuberculosis. J Immunol 182: 3757-3767.

Medina E, North RJ. 1998. Resistance ranking of some common inbreb mouse strains to Mycobacterium tuberculosis and realtionship to major histocompatibiltiy complex haplotype and Nramp1 genotype. Immunology 93: 270-274.

Medina E, North R. 1999. Genetically susceptible mice remain proportionally more susceptible to tuberculosis af ter vaccination. Immunology 96: 16-21.

Mishra BB, Rathinam VAK, Martens GW, Martinot AJ, Kornfeld H, Fitzgerald KA, Sassetti CM. 2013. Nitric oxide controls the immunopathology of tuberculosis by inhibiting NLRP3 inflammasome-dependent processing of IL-1 $\beta$. Nat Immunol 14: 52-60.

Nandi B, Behar SM. 2011. Regulation of neutrophils by interferon- $\gamma$ limits lung inflammation during tuberculosis infection. J Exp Med 208: 2251-2262.

Pan H, Yan B, Rojas M, Shebzukhov Y, Zhou H, Kobzik L, Higgins D, Daly M, Bloom B, Kramnik I. 2005. Ipr1 gene mediates innate immunity to tuberculosis. Nature 434: 767-772.

Pearl JE, Saunders BM, Ehlers S, Orme IM, Cooper AM. 2001. Inflammation and lymphocyte activation during mycobacterial infection in the interferon- $\gamma$-deficient mouse. Cell Immunol 211: 43-50.

Pearl JE, Torrado E, Tighe M, Fountain JJ, Solache A, Strutt T, Swain S, Appelberg R, Cooper AM. 2012. Nitric oxide inhibits the accumulation of $\mathrm{CD} 4{ }^{+} \mathrm{CD} 44^{\text {hi }}$ Tbet $^{+} \mathrm{CD} 69^{\text {lo }}$ $\mathrm{T}$ cells in mycobacterial infection. Eur J Immunol 42: 3267-3279.

Pichugin AV, Yan B-S, Sloutsky A, Kobzik L, Kramnik I. 2009. Dominant role of the sst 1 locus in pathogenesis of necrotizing lung granulomas during chronic tuberculosis infection and reactivation in genetically resistant hosts. Am J Pathol 174: 2190-2201.

Reiley W, Calayag M, Wittmer S, Huntington J, Pearl J, Fountain J, Martino C, Roberts A, Cooper A, Winslow G, et al. 2008. ESAT-6-specific CD4 T cell responses to aerosol Mycobacterium tuberculosis infection are initiated in mediastinal lymph nodes. Proc Natl Acad Sci 105: 10961-10966.

Reiley WW, Shafiani S, Wittmer ST, Tucker-Heard G, Moon JJ, Jenkins MK, Urdahl KB, Winslow GM, Woodland DL. 2010. Distinct functions of antigen-specific CD4 T cells during murine Mycobacterium tuberculosis infection. Proc Natl Acad Sci 107: 19408-19413.

Rhoades ER, Frank AA, Orme IM. 1997. Progression of chronic pulmonary tuberculosis in mice aerogenically infected with virulent Mycobacterium tuberculosis. Tuber Lung Dis 78: 57-66.

Robinson R, Khader S, Martino C, Fountain J, TeixeiraCoelho M, Pearl J, Smiley S, Winslow G, Woodland D, Walter M, et al. 2010. Mycobacterium tuberculosis infection induces ill2rb1 splicing to generate a novel IL12R $\beta 1$ isoform that enhances DC migration. J Exp Med 207: 591-605.

Saito M, Iwawaki T, Taya C, Yonekawa H, Noda M, Inui Y, Mekada E, Kimata Y, Tsuru A, Kohno K. 2001. Diphtheria toxin receptor-mediated conditional and targeted cell ablation in transgenic mice. Nat Biotechnol 19: 746-750. 
A.M. Cooper

Samstein M, Schreiber HA, Leiner IM, Sušac B, Glickman MS, Pamer EG, Lanzavecchia A. 2013. Essential yet limited role for $\mathrm{CCR}^{+}$inflammatory monocytes during Mycobacterium tuberculosis-specific T cell priming. eLife 2: $\mathrm{e} 01086$.

Sánchez F, Radaeva TV, Nikonenko BV, Persson A-S, Sengul S, Schalling M, Schurr E, Apt AS, Lavebratt C. 2003. Multigenic control of disease severity after virulent $M y$ cobacterium tuberculosis infection in mice. Infect Immun 71: $126-131$.

Shizuru J, Jerabek L, Edwards C, Weissman I. 1996. Transplantation of purified hematopoietic stem cells: Requirements for overcoming the barriers of allogeneic engraftment. Biol Blood Marrow Transplant 2: 3-14.

Slight SR, Rangel-Moreno J, Gopal R, Lin Y, Fallert Junecko BA, Mehra S, Selman M, Becerril-Villanueva E, BaqueraHeredia J, Pavon L, et al. 2013. CXCR5 ${ }^{+}$T helper cells mediate protective immunity against tuberculosis. J Clin Invest 123: 712-726.
Sprent J. 2013. The power of dilution: Using adoptive transfer to study TCR transgenic T cells. J Immunol 191: 53255326.

Srivastava S, Ernst JD. 2013. Cutting edge: Direct recognition of infected cells by CD4 T cells is required for control of intracellular Mycobacterium tuberculosis in vivo. J Immunol 191: 1016-1020.

Torrado E, Fountain JJ, Robinson RT, Martino CA, Pearl JE, Rangel-Moreno J, Tighe M, Dunn R, Cooper AM. 2013. Differential and site specific impact of B cells in the protective immune response to Mycobacterium tuberculosis in the mouse. PLoS ONE 8: e61681.

Wolf AJ, Linas B, Trevejo-Nunez GJ, Kincaid E, Tamura T, Takatsu K, Ernst JD. 2007. Mycobacterium tuberculosis infects dendritic cells with high frequency and impairs their function in vivo. J Immunol 179: 2509-2519.

Wolf A, Desvignes L, Linas B, Banaiee N, Tamura T, Takatsu K, Ernst J. 2008. Initiation of the adaptive immune response to Mycobacterium tuberculosis depends on antigen production in the local lymph node, not the lungs. J Exp Med 205: 105-115. 


\section{$\&_{\mathrm{CSH}}^{\infty} \&$ Cold Spring Harbor

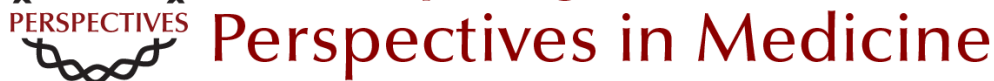

\section{Mouse Model of Tuberculosis}

Andrea M. Cooper

Cold Spring Harb Perspect Med 2015; doi: 10.1101/cshperspect.a018556 originally published online September 25, 2014

\section{Subject Collection Tuberculosis}

Transmission and Institutional Infection Control of Tuberculosis Edward A. Nardell

Innate and Adaptive Cellular Immune Responses

to Mycobacterium tuberculosis Infection Katrin D. Mayer-Barber and Daniel L. Barber

Tuberculosis Comorbidity with Communicable and Noncommunicable Diseases

Matthew Bates, Ben J. Marais and Alimuddin Zumla

Host-Directed Therapies for Tuberculosis David M. Tobin

Immunity and Immunopathology in the

Tuberculous Granuloma

Antonio J. Pagán and Lalita Ramakrishnan

Tuberculosis Drug Development: History and

Evolution of the Mechanism-Based Paradigm? Sumit Chakraborty and Kyu Y. Rhee

Genetic Approaches to Facilitate Antibacterial

Drug Development

Dirk Schnappinger

The Tuberculosis Drug Discovery and

Development Pipeline and Emerging Drug Targets

Khisimuzi Mdluli, Takushi Kaneko and Anna Upton
Clinical Aspects of Adult Tuberculosis Robert Loddenkemper, Marc Lipman and Alimuddin Zumla

Advances in Diagnostic Assays for Tuberculosis Stephen D. Lawn

Diagnosis and Management of Latent

Tuberculosis Infection

Laura Muñoz, Helen R. Stagg and Ibrahim

Abubakar

Mycobacterial Growth

Iria Uhía, Kerstin J. Williams, Vahid Shahrezaei, et al.

Multidrug-Resistant Tuberculosis and Extensively

Drug-Resistant Tuberculosis

Kwonjune J. Seung, Salmaan Keshavjee and Michael L. Rich

The Mycobacterial Cell Wall--Peptidoglycan and

Arabinogalactan Luke J. Alderwick, James Harrison, Georgina S. Lloyd, et al.

Tuberculosis and HIV Coinfection Judith Bruchfeld, Margarida Correia-Neves and Gunilla Källenius

Imaging in Tuberculosis Jamshed B. Bomanji, Narainder Gupta, Parveen Gulati, et al.

For additional articles in this collection, see http://perspectivesinmedicine.cshlp.org/cgi/collection/ 